\title{
Endoscopic Management of Double Esophageal Perforation by Ingested Foreign Body Using Over-the-Scope Clip: A Case Report
}

\author{
Prasanta Debnath ${ }^{1} \quad$ Pravin Rathi $^{1} \quad$ Sujit Nair ${ }^{1}$ \\ ${ }^{1}$ Department of Gastroenterology, Topiwala National Medical \\ College and Bai Yamunabai Laxman Nair Charitable Hospital, \\ Mumbai, Maharashtra, India
}

J Digest Endosc 2020;11:153-155

\begin{abstract}
Address for correspondence Prasanta Debnath, MD, Department of Gastroenterology, Topiwala National Medical College and Bai Yamunabai Laxman Nair Charitable Hospital, Room no 717, 7th floor, OPD Building, Dr A.L Nair Road, Mumbai Central, Mumbai 400008, Maharashtra, India (e-mail: prasantad89@gmail.com).
\end{abstract}

\author{
Abstract \\ Keywords \\ - esophageal foreign \\ body \\ - endoprosthesis \\ - endoscopy \\ - esophageal \\ perforation
}

Esophageal perforation is a life-threatening condition with a high mortality rate. First described around 300 years ago, management of this fatal condition has emerged from surgical to endoscopic modalities with much less morbidity and mortality when instituted early. We present this case of 55-year-old male, with double esophageal perforation by meat bone, perforating lower esophageal wall, leading to localized hydropneumothorax on right side with mild bilateral pleural effusion managed endoscopically with Over-the-Scope-Clip. Endoscopic management of esophageal perforation has been well mentioned in literature, without any mention of such management in case of double esophageal perforation. Surgery with or without endoscopy remains the main stay of management of such cases.

\section{Introduction}

Esophageal perforation (EP) though rare, is a potentially life-threatening event. In 1724, Hermann Boerhaave reported the first case of spontaneous esophageal rupture after repeated episodes of vomiting. ${ }^{1}$ The first attempt at surgical repair was done by Barrett and Olson in 1947.2,3 With the development of endoscopic technology during the last two decades, endoscopic clips and self-expanding stents have been used successfully and widely in the treatment of esophageal perforations. ${ }^{4,5}$ Esophageal injury was first closed endoscopically with the placement of clips in $1995 .{ }^{6}$ To date, the method has been successful, especially in the treatment of small $(<2 \mathrm{~cm})$ injuries. EP is most commonly iatrogenic following instrumentation, followed by spontaneous or caused by foreign body ingestion, thoracic trauma, or malignant growth. A total of $80 \%$ of cervical perforations and for 9 to $35 \%$ of all esophageal perforations are secondary to ingested foreign bodies. ${ }^{7}$

With a rise in interventional endoscopic techniques, notably natural orifice transluminal endoscopic surgery (NOTES), endoscopic submucosal dissection (ESD), peroral endoscopic myotomy (POEM), and endoscopic full-thickness resection
(EFTR) for the management of various benign and malignant conditions of the gastrointestinal (GI) tract, all have contributed to the rising incidence of iatrogenic GI tract disruptions. Foreign body impacting at narrowed portion can lead to its extraluminal migration perforating nearby organs, trachea or aorta, often with fatal consequences, or mediastinitis which spreads easily as the esophagus is surrounded by loose stromal connective tissue. ${ }^{8}$

Diagnosis of EP requires radiologic and endoscopic examination. Conventional radiology can show various complications like pneumothorax, pneumoperitoneum, pneumomediastinum, subcutaneous emphysema. A computed tomography (CT) scan can help in confirming the diagnosis. Endoscopy with a sensitivity and specificity of 100 and $83 \%$, respectively, can be diagnostic and therapeutic at the same setting, enabling the extraction of the foreign body. Repair of perforation can be considered endoscopically using endoprosthesis, endoscopic clipping, or gluing. If endoscopy fails surgical intervention for foreign body removal and perforation, closure is to be considered. However, the choice of an appropriate treatment depends on the type of perforation, the performance status of the patient, and the availability of resources. 


\section{Case Report}

A 55-year-old male patient presented with complaints of sudden onset dysphagia with odynophagia for 2 days before presenting to emergency department of our hospital. Symptoms has started soon after his night meal in which he had chicken which was swallowed improperly. No history of fever, cough or shortness of breath was there and he was hemodynamically stable. There was no history of alcohol intoxication or substance abuse. Before coming to our center, he visited a nearby hospital where chest radiography along with CT thorax with abdomen was done. CT examination was suggestive of linear hyperdense (density, 243-296 HU) lesion of size $3.1 \mathrm{~cm}$ in lower esophagus with localized pocket of air fluid level of $1.8 \mathrm{~cm}$ thickness and $7 \mathrm{~cm}$ in craniocaudal extent on right side; along with mild bilateral pleural effusion ( - Fig. 1A). However, in view of nonavailability of endoscope he was referred to our hospital. On examination, there was local tenderness in subxiphoid and epigastric region. His routine investigations were normal. Patient was started on intravenous antibiotics. Upper GI endoscopy (UGIE) was performed which was suggestive of foreign body, most probably chicken bone in lower esophagus which was impacted in one wall of the esophagus with free perforation seen on the opposite wall $(\boldsymbol{- F i g}$. 1B). The bone was held carefully with Olympus Rat Tooth Alligator
Jaw Grasping Forceps and was gently removed (-Fig. 2A). Post removal, endoscopy revealed presence of two fistulous tracks in both lateral walls of esophagus of size $1.5 \times 0.5 \mathrm{~cm}$ in in one side and $1 \times 0.5 \mathrm{~cm}$ on the other side ( - Fig. 2B). The edges of the large fistula were ablated with argon plasma coagulation. Over-the-Scope-Clip (OTSC, Ovesco Endoscopy $\mathrm{GmBH}$, Tubingen, Germany) was used to seal off the perforation. The target area was approached perpendicularly, facing the lesion, and the two wound edges were sucked into the cap resulting in a full-thickness clip apposition ( - Fig. 3). The other fistula could not be approached in the same setting because of marked surrounding edema and putting a second clip in the same setting, might lead to mark esophageal luminal compromise. Under endoscopic guidance, guidewire was passed to antrum and nasojejunal tube was inserted for feeding. Intravenous antibiotics was continued for 7 days. Patient was discharged in hemodynamically stable condition without any fresh complaints. Repeat UGIE was considered after 3 months. Edema around the fistula had substantially resolved by that time and repeat procedure using Overthe-Scope Clip (OTSC, Ovesco Endoscopy GmBH, Tubingen, Germany) was considered for the second fistula (-Fig. 4). Similar to previous procedure, argon plasma coagulation was considered to ablate the edges first followed by application of the clip. Patient tolerated procedure well.

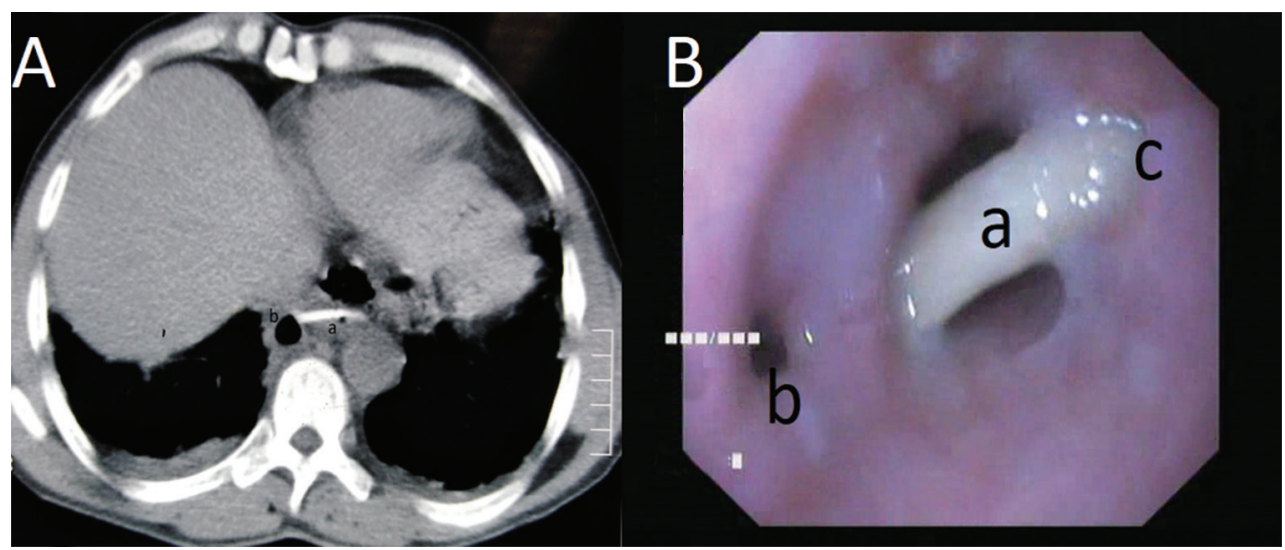

Fig. 1 (A) CT scan showing impacted meat (chicken) bone (a) and localized air collection in right side (b). (B) Endoscopic view: impacted Meat (chicken) bone (a); and perforation sites in lateral walls of esophagus (b and c).

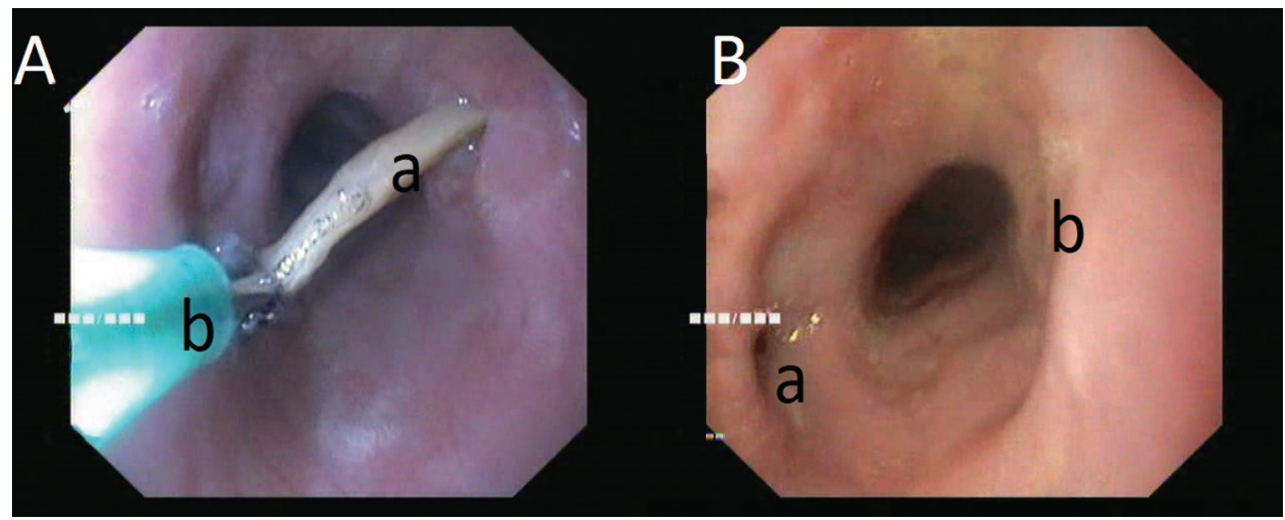

Fig. 2 (A) Endoscopic removal of meat bone (a) with Olympus Rat Tooth Alligator Jaw Grasping Forceps (b). (B) Endoscopic view of (a) perforation sites and (b) below the esophageal fold in two opposite walls of esophagus. 


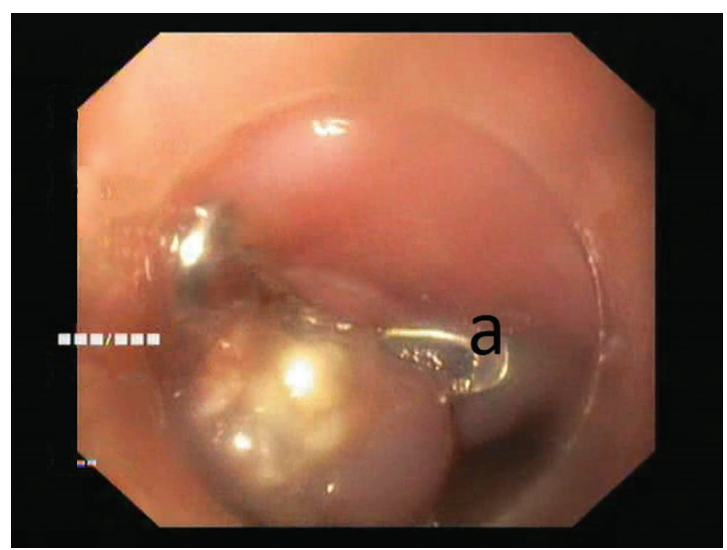

Fig. 3 Endoscopic Over-The-Scope Clip application in perforation site on right lateral wall of esophagus (a).

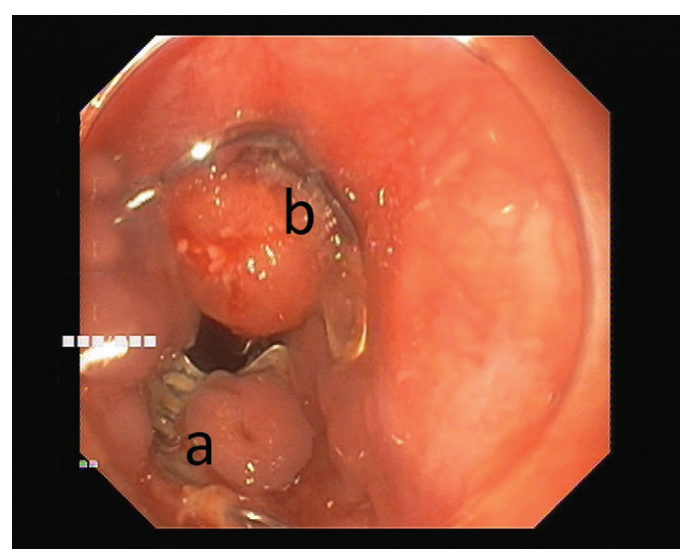

Fig. 4 Endoscopic Over-The-Scope Clip application right lateral wall (a) left lateral wall (b) of esophagus.

\section{Discussion}

To our knowledge, this remains the first case of double esophageal perforation with localized right hydropneumothorax, who presented after 24 hours, managed successfully with endoscopic procedure using OTSC. OTSC can provide full-thickness closure of open defects up to 2 to $3 \mathrm{~cm}$. Three different clip tooth shapes are available which are suitable for different indications: $(\mathrm{T})$ traumatic, for fistulas or perforations (A) atraumatic, to control bleeding, and (GC) gastrostomy closure. Multicenter prospective cohort study from Europe with iatrogenic perforations (colon being the most common site, followed by duodenal, gastric, and esophageal cases), has shown that $89 \%$ of patients had successful closures after OTSC application without any adverse events. ${ }^{9}$ Singlecenter Swiss study has shown that OTSC used for fistulae and anastomotic leakage after GI surgery, primary technical success, defined as the adequate deployment of the OTSC on the target lesion, and clinical success, defined as resolution of the problem of 85 and $67 \%$, respectively..$^{10}$ Multicentric study at Italy has shown that OTSC used in iatrogenic perforation during diagnostic or therapeutic endoscopy had technical success rate of $10 \%$ with clinical success of $90 \%{ }^{11}$ German study has shown that in a set of nonsurgical perforation and postoperative leak or perforation, successful closure using OTSC was obtained in $76.5 \%$ of patients, which had resulted in a significant shorter hospital stay. ${ }^{12}$

Multidisciplinary approach (surgeon, endoscopy specialist, and intensive care therapist) should be considered in management of esophageal perforations. Endoscopic closure of early, well-defined esophageal perforations represents a therapeutic alternative to surgical treatment thereby reducing morbidity and even mortality to a large extent.

\section{Financial Support}

Nil.

\section{Conflict of Interest}

None.

\section{References}

1 Derbes VJ, Mitchell RE Jr. Hermann Boerhaave's Atrocis, nec descripti prius, morbi historia, the first translation of the classic case report of rupture of the esophagus, with annotations. Bull Med Libr Assoc 1955;43(2):217-240

2 Barrett NR, Franklin RH. Concerning the unfavourable late results of certain operations performed in the treatment of cardiospasm. Br J Surg 1949;37(146):194-202, illust

3 Olsen AM, Clagett OT. Spontaneous rupture of the esophagus; report of a case with immediate diagnosis and successful surgical repair. Postgrad Med 1947;2(6):417-421

4 Fischer A, Thomusch O, Benz S. von Dobschuetz E, Baier P, Hopt UT. Nonoperative treatment of 15 benign esophageal perforations with self-expandable covered metal stents. Ann Thorac Surg 2006;81(2):467-472

5 Johnsson E, Lundell L, Liedman B. Sealing of esophageal perforation or ruptures with expandable metallic stents: a prospective controlled study on treatment efficacy and limitations. Dis Esophagus 2005;18(4):262-266

6 Wewalka FW, Clodi PH, Haidinger D. Endoscopic clipping of esophageal perforation after pneumatic dilation for achalasia. Endoscopy 1995;27(8):608-611

7 Chirica M, Champault A, Dray X, et al. Esophageal perforations. J Visc Surg 2010;147(3):e117-e128

8 Champault A, Cattan P. [Aortic rupture due to a swallowed medical needle] (in French)J Chir (Paris) 2009;146(4):442-443

9 Voermans RP, Le Moine O, von Renteln D, et al; CLIPPER Study Group. Efficacy of endoscopic closure of acute perforations of the gastrointestinal tract. Clin Gastroenterol Hepatol 2012;10(6):603-608

10 Sulz MC, Bertolini R, Frei R, Semadeni GM, Borovicka J, Meyenberger C. Multipurpose use of the over-the-scope-clip system ("Bear claw") in the gastrointestinal tract: Swiss experience in a tertiary center. World J Gastroenterol 2014;20(43): 16287-16292

11 Mangiavillano B, Caruso A, Manta R, et al. Over-the-scope clips in the treatment of gastrointestinal tract iatrogenic perforation: A multicenter retrospective study and a classification of gastrointestinal tract perforations. World J Gastrointest Surg 2016;8(4):315-320

12 Raithel M, Albrecht H, Scheppach W, et al. Outcome, comorbidity, hospitalization and 30-day mortality after closure of acute perforations and postoperative anastomotic leaks by the over-the-scope clip (OTSC) in an unselected cohort of patients. Surg Endosc 2017;31(6):2411-2425 